\title{
Estrategias de afrontamiento utilizadas por los pacientes con tuberculosis de un centro de salud
}

\author{
Belito-de la Cruz Hildaa ${ }^{a}$ Fernández-Cruzado Valeriaa ${ }^{a}, S^{\prime}$ chez-Gutiérrez Shirleya ${ }^{\text {, Farro-Peña Gianina }}{ }^{1, b, c}$
}

\section{RESUMEN}

Cuando una persona recibe el diagnóstico de una enfermedad crónica como la tuberculosis cambia la forma como se ve a sí misma y a su vida, ello afecta profundamente el autoconcepto, y la adaptación dependerá de una multiplicidad de factores, como la edad, el sexo, el estatus social, el apoyo, la educación, las creencias religiosas, los patrones de personalidad, la inteligencia, los estilos de afrontamiento, el equilibrio o control emocional, etc. Objetivo: identificar las estrategias de afrontamiento utilizadas por los pacientes con tuberculosis. Material y métodos: estudio descriptivo de corte transversal. Se realizó en el Centro de Salud «Santa Rosa» de San Juan de Lurigancho. El cuestionario Brief Cope fue aplicado a 30 pacientes. Para su validación, fue sometido a juicio de expertos y a una prueba piloto. Resultados: el 86,6\% de la población utiliza el afrontamiento activo, el 73,3\% de la población siempre utiliza la aceptación y el 53,3\% utiliza la autodistracción como medio de afrontamiento. Conclusiones: de la dimensión estrategias centradas en el problema, los pacientes que asisten a la ESN-PCT del Centro de Salud Santa Rosa utilizan, en mayor porcentaje, el afrontamiento activo.

Palabras clave: estrategias, afrontamiento, tuberculosis pulmonar. Perú (Fuente DeCs BIREME).

\section{Ways of coping used by patients with tuberculosis of a community center}

\begin{abstract}
When a patient is diagnosed with chronic tuberculosis, the way the person sees his or her life changes. Auto concept is affected and the adaptation will depend on multiple factors such as age, gender, social status, education, religious believes, personality, intelligence, ways of coping, self control, etc. Objetive: The aim of the project was to identify the ways of coping in patients with Tuberculosis. Material and Methods: A descriptive and cross-sectional research was conducted at «Santa Rosa» community center in San Juan de Lurigancho. The ways of coping questionnaire was applied to a sample of 30 patients with tuberculosis. An expert review and pilot study were conducted to assess the credibility and validity of the instrument. Statistical analysis of the quantitative data was conducted. Results: The findings of the study revealed that $86.6 \%$ of the interviewers use active coping, $73.3 \%$ of the population always uses the acceptance and $53.3 \%$ use self-distraction as a means of confrontation. Conclusions. Patients who attend the ESN-PCT at «Santa Rosa» community center mostly use problem focused ways of coping.
\end{abstract}

Key words: Coping strategies, cope, tuberculosis. Peru (Source DeCs BIREME).

\section{INTRODUCCIÓN}

En el Perú y el mundo la tuberculosis es considerada uno de los principales problemas de salud pública más prevalente y de gran impacto a nivel físico, psicológico, social y económico. En algunos países se está producien- do una disminución considerable de los casos, pero en otros el descenso está sucediendo más lentamente.

A pesar de esto, la situación en el Perú sigue siendo de cuidado ya que 34811 personas fueron afectadas por esta enfermedad, además, se sabe que el $75 \%$ de las infeccio-

${ }^{1}$ Facultad de Enfermería,Universidad Peruana Cayetano Heredia. Lima,Perú.

${ }^{a}$ Licenciada en Enfermeria; ${ }^{b}$ Magíster en Salud Pública ; ${ }^{\mathrm{c}}$ Profesora principal. 
nes y muertes por tuberculosis ocurren entre los 15 y los 54 años de edad, el grupo de población económicamente más productivo. El panorama en el interior del país muestra que los departamentos registrados con mayor tasa de incidencia en tuberculosis son: Loreto, La Libertad, Madre de Dios, Tacna, Ica, Arequipa y Junín. Cabe recordar que en la actualidad esta enfermedad ha desarrollado formas cada vez más resistentes al tratamiento, es así como Lima y Callao concentran aproximadamente el 58\% de todos los casos de tuberculosis y el $82 \%$ de tuberculosis multidrogorresistente (1).

De esta manera, se puede afirmar que no existe distrito en Lima que esté libre de la tuberculosis. Según datos del Ministerio de Salud, en cuatro distritos del cono este de Lima se han reportado, el año pasado, 2265 casos nuevos de tuberculosis pulmonar, siendo San Juan de Lurigancho el distrito que presenta el mayor número de pacientes con esta enfermedad. De acuerdo con las cifras de la Estrategia Sanitaria Regional de Tuberculosis de la DISA IV, este último distrito reportó 1148 casos (2).

A pesar de estos datos alarmantes, uno de los aspectos más importantes y fascinantes en relación con la tuberculosis, es que es una enfermedad $100 \%$ curable y prevenible; sin embargo, se ha convertido en la infección trasmisible más importante en los seres humanos. Múltiples factores explican esta situación, sobre todo factores demográficos socioeconómicos que favorecen las migraciones, la presencia de determinantes sociales y estilos de vida inadecuados, la escasa atención prestada al control de la tuberculosis en muchos países y la peligrosa comorbilidad con la epidemia del VIH, además que algunas de las fuentes de contagio con frecuencia quedan sin ser diagnosticadas y sin recibir tratamiento, pero lo más grave aun es el hecho de que cuando lo reciben, la prescripción inadecuada y la falta de adherencia al tratamiento constituye un problema complejo y multifactorial que favorece un aumento de la tuberculosis (TB) y la tuberculosis multidrogorresistente (3).

Cuando una persona recibe el diagnóstico de una enfermedad crónica como la tuberculosis cambia la forma como se ve a sí misma y a su vida, afectando profundamente el autoconcepto; su adaptación dependerá de una multiplicidad de factores, como la edad, el sexo, el estatus social, el apoyo, la educación, las creencias religiosas, los patrones de personalidad, la inteligencia, los estilos de afrontamiento, el equilibrio o control emocional, etc. Igualmente, estas aparecen, se mantienen o se agravan en función de las conductas individuales; por lo tanto, es fundamental evaluar el concepto de estilos de afrontamiento para enten- der las estrategias que pone en acción o en marcha una persona cuando se enfrenta a la tuberculosis, dado que de esto dependerá el éxito o fracaso del tratamiento.

El concepto de estrategias de afrontamiento comienza a tener importancia en los años 1940 y 1950 con las que se busca que la persona que está frente a una situación estresante tenga la capacidad de enfrentar y llevar mejor la enfermedad por medio de recursos o elementos que le permiten llegar a un objetivo final (4). Se definen como un conjunto de esfuerzos cognitivos y conductuales que realiza una persona para controlar (minimizar, dominar, tolerar) las demandas (externas, internas y su posible conflicto) generadoras de estrés, que son evaluadas como excedentes o desbordantes de los recursos del individuo (5). El objetivo de las estrategias de afrontamiento es analizar cómo las personas se enfrentan a elementos estresantes determinados: enfermedades crónicas, estresores cotidianos o sucesos vitales estresantes.

Carver, considerando el enfoque de Lazarus y Folkman, señala tres escalas que evalúan los aspectos: centrados en la solución del problema, donde la persona hace frente a su problema manejando sus consecuencias y adaptándose a la nueva situación de salud, afrontamiento de tipo emocional en el cual reduce los sentimientos negativos manteniendo el optimismo y la esperanza frente al proceso de la enfermedad y respuestas menos funcionales donde valora la manera que su nuevo estresor resulte menos desagradable. De esta manera, además de redefinir en tres grandes aspectos el tipo de afrontamiento, Carver aporta una metodología que permite estudiar con mayor precisión las conductas, tanto cognitivas como motrices, que utilizan las personas ante el estrés (4). Si bien es cierto que este concepto se ha desarrollado ampliamente en otras áreas, para la enfermería el afrontamiento es clave para la teoría e investigación en adaptación y salud. La North American Nursing Diagnosis Association (NANDA) define el afrontamiento como procesos cognitivos y conductuales para el manejo de problemas o agentes estresantes actuales o futuros, e identifica este concepto como un diagnóstico de enfermería; Carpenito lo define como el manejo de los factores de estrés internos o externos dirigidos hacia la adaptación (5).

La importancia que la Enfermería como ciencia que se centra en el cuidado holístico de los individuos en presencia o ausencia de enfermedad, con calidad y calidez, aborde temas como este, radica en que el profesional enfermero forma parte del equipo multidisciplinario de salud (6), de los programas dirigidos al control, prevención, tratamiento de tuberculosis, y es quien dirige los procesos de atención 
que se brinda como: la terapia directamente observada de corta duración (DOTS), las visitas domiciliarias, las charlas educativas, y la entrevista de enfermería, constituye así un eje fundamental de acción de la estrategia (6); tanto por su labor educativa como por el rol orientador que debe brindar, dado que un individuo, al ser diagnosticado de una enfermedad como la tuberculosis, adopta diversos mecanismos de defensa ante una situación nueva, a esto se suma el desconocimiento sobre su enfermedad, el tratamiento farmacológico, todo ello conllevaría a muchos riesgos, entre ellos el abandono del tratamiento. Las investigadoras se plantean el desarrollo de la presente investigación reconociendo la importancia del proceso de afrontamiento de los pacientes con tuberculosis, con la finalidad de que la información obtenida pueda ser utilizada por el personal del Centro de Salud «Santa Rosa» de San Juan de Lurigancho.

\section{MATERIAL Y MÉTODOS}

Se utilizó el método descriptivo ya que se detalla cuáles son las estrategias de afrontamiento utilizadas por los pacientes con TBC; es de corte transversal porque se conoció estas estrategias haciendo un corte en el tiempo en un determinado momento. Se realizó en el Centro de Salud «Santa Rosa» en el programa de prevención y control de tuberculosis ubicado en el distrito con mayor prevalencia de pacientes diagnosticados con tuberculosis, San Juan de Lurigancho. La población estuvo constituida por 30 pacientes, pertenecientes a la ESN - PCT del Centro de Salud «Santa Rosa» de San Juan de Lurigancho en el periodo diciembre 2013 - enero 2014.

La técnica que se utilizó fue la encuesta y el instrumento un cuestionario. Como referencia para la elaboración del instrumento se tomó el cuestionario Brief Cope, versión abreviada de la escala multidimensional de evaluación de los estilos de afrontamiento (COPE) creado en 1989 por Carver. El Brief Cope (1997). Esta versión abreviada omite dos subescalas del COPE completo (supresión de actividades distractoras y refrenar el afrontamiento) ya que no fue confirmada su validez en los trabajos previos. Otras tres subescalas fueron ligeramente reenfocadas debido a que habían demostrado ser problemáticas en los estudios precedentes. A la subescala originalmente denominada reinterpretación positiva y crecimiento, se le denomina solamente reinterpretación positiva. Finalmente, la subescala llamada humor, no forma parte del inventario original COPE y fue añadida por Carver, porque se consideró que era evidente la importancia de esta respuesta (7). Su creador considera que es idóneo en investigación, ya que proporciona una medida breve de afrontamiento.
Fue desarrollado con la intención de reducir la sobrecarga y el esfuerzo que tenían que realizar al responder al inventario completo. Escogió dos ítems por escala, aquellos que habían tenido un peso mayor en análisis factorial previo, por la claridad, y por el sentido del ítem para los respondientes. El Brief Cope posee propiedades psicométricas satisfactorias: todas las escalas demostraron buenos índices de consistencia interna (coeficiente alfa de Cronbach entre 0,60 y 0,90) (7).

El cuestionario consta de dos partes:

Primera parte: contiene los datos sociodemográficos tales como sexo, edad cronológica, ocupación y grado de instrucción, tiempo de enfermedad y tiempo de tratamiento.

Segunda parte: contiene tres dimensiones compuestas por 14 subescalas, cada una con dos ítems, que hacen un total de 28 preguntas. La dimensión centrada en el problema consta de dos subescalas: afrontamiento activo y planificación; la dimensión centrada en la emoción consta de cinco subescalas: apoyo instrumental, uso de apoyo emocional, religión, reinterpretación positiva y aceptación; la dimensión centrada en la emoción menos funcional consta de siete subescalas: autoinculpación, autodistracción, negación, desconexión conductual, desahogo, uso de sustancias y humor.

Las preguntas fueron elaboradas sobre la base de la versión disposicional del instrumento, la cual no tiene respuestas correctas ni incorrectas y se toma lo que cada persona suele hacer con frecuencia, además, se consideró el emparejamiento del contenido del ítem con la subescala que se quiere evaluar. Para determinar la validez del instrumento se procedió a realizar un juicio de expertos en el que participaron: 1 médico, 4 enfermeras y 3 psicólogas, mediante la prueba binomial, para la confiabilidad se empleó el análisis alfa de Cronbach que evalúa la homogeneidad interna.

Para iniciar la recolección de datos una vez aprobado el proyecto por las instancias de la Universidad Peruana Cayetano Heredia y la Facultad de Enfermería, se realizaron las coordinaciones con el jefe del Centro de Salud «Santa Rosa» de San Juan de Lurigancho para abordar a los pacientes. Las investigadoras, en un primer momento, captaron a la población un determinado día en el cual se les explicó en qué consistía el estudio. A los pacientes que aceptaron participar voluntariamente se les hizo entrega del consentimiento informado; una vez firmado dicho documento se coordinó con ellos una fecha y la hora para que pudieran resolver el cuestionario. 


\section{RESULTADOS}

Tabla 1. Estrategias de afrontamiento centrado en el problema utilizadas por los pacientes con tuberculosis de un centro de salud

\begin{tabular}{|c|c|c|c|c|c|c|c|c|c|c|}
\hline \multirow{2}{*}{$\begin{array}{l}\text { Afrontamien to centrado } \\
\text { en el problema }\end{array}$} & \multicolumn{2}{|c|}{ Nunca } & \multicolumn{2}{|c|}{ Casi nunca } & \multicolumn{2}{|c|}{$\begin{array}{c}\text { Casi } \\
\text { siempre }\end{array}$} & \multicolumn{2}{|c|}{ Siempre } & \multicolumn{2}{|c|}{ Total } \\
\hline & $\mathbf{N}$ & $\%$ & $\mathbf{N}$ & $\%$ & $\mathbf{N}$ & $\%$ & $\mathbf{N}$ & $\%$ & $\mathbf{N}$ & $\%$ \\
\hline \multicolumn{11}{|l|}{ Afrontamiento Activo } \\
\hline $\begin{array}{l}\text { Concentro mis e suerzos en } \\
\text { hacer algo sobre mi } \\
\text { enfermedad }\end{array}$ & 0 & 0 & 0 & 0 & 4 & 13,3 & 26 & 86,6 & 30 & 100 \\
\hline $\begin{array}{l}\text { Tomo medidas para intentar } \\
\text { mejorar mi enfermedad }\end{array}$ & 0 & 0 & 0 & 0 & 4 & 13,3 & 26 & 86,6 & 30 & 100 \\
\hline \multicolumn{11}{|l|}{ Planificación } \\
\hline $\begin{array}{l}\text { estrategia sobre cómo } \\
\text { cuidarme ahora que estoy } \\
\text { enfermo }\end{array}$ & 0 & 0 & 1 & 3,3 & 11 & 36,6 & 18 & 60 & 30 & 10 \\
\hline $\begin{array}{l}\text { Pienso deteni damente sobre } \\
\text { las acciones a seguir } \\
\text { relacionados con mi } \\
\text { enfermedad }\end{array}$ & 1 & 3,3 & 2 & 6,6 & 9 & 30 & 18 & 60 & 30 & 100 \\
\hline
\end{tabular}

Tabla 2. Estrategias de afrontamiento centrado en la emoción utilizadas por los pacientes con tuberculosis de un centro de salud

\begin{tabular}{|c|c|c|c|c|c|c|c|c|c|c|}
\hline \multirow{2}{*}{$\begin{array}{l}\text { Afrontamiento centrado en la } \\
\text { emoción }\end{array}$} & \multicolumn{2}{|c|}{ Nunca } & \multicolumn{2}{|c|}{ Casinunca } & \multicolumn{2}{|c|}{ Casi siempre } & \multicolumn{2}{|c|}{ Siempre } & \multicolumn{2}{|c|}{ Total } \\
\hline & $\mathbf{N}$ & $\%$ & $\mathbf{N}$ & $\%$ & $\mathbf{N}$ & $\%$ & $\mathrm{~N}$ & $\%$ & $\mathbf{N}$ & $\%$ \\
\hline \multicolumn{11}{|l|}{ Apoyo instrum ental } \\
\hline $\begin{array}{l}\text { Intento conseguir que alguien } \\
\text { me ayude o aconseje sobre mi } \\
\text { enferm edad }\end{array}$ & 7 & 23,3 & 5 & 16,6 & 10 & 33,3 & 8 & 26,6 & 30 & 100 \\
\hline $\begin{array}{l}\text { Consigo que otras per sonas me } \\
\text { ayuden o aconsejen sobre mi } \\
\text { enferm edad }\end{array}$ & 6 & 20,0 & 4 & 13,3 & 15 & 50,0 & 5 & 16,6 & 30 & 100 \\
\hline \multicolumn{11}{|l|}{ Uso de apoyo emocional } \\
\hline $\begin{array}{l}\text { Consigo apoyo emocional de } \\
\text { otras personas }\end{array}$ & 7 & 23,3 & 2 & 6,6 & 8 & 26,6 & 13 & 43,3 & 30 & 100 \\
\hline $\begin{array}{l}\text { Consigo el consuelo yla } \\
\text { comprensión de otras personas }\end{array}$ & 8 & 26,6 & 5 & 16,6 & 8 & 26,6 & 9 & 30,0 & 30 & 100 \\
\hline
\end{tabular}

Tabla 2.1. Estrategias de afrontamiento centrado en la emoción utilizadas por los pacientes con tuberculosis de un centro de salud

\begin{tabular}{|c|c|c|c|c|c|c|c|c|c|c|}
\hline \multirow{2}{*}{$\begin{array}{l}\text { Afrontamiento centrado } \\
\text { en la emoción }\end{array}$} & \multicolumn{2}{|c|}{ Nunca } & \multicolumn{2}{|c|}{ Casi nunca } & \multicolumn{2}{|c|}{ Casi siempre } & \multicolumn{2}{|c|}{ Siempre } & \multicolumn{2}{|c|}{ Total } \\
\hline & $\mathbf{N}$ & $\%$ & $\mathbf{N}$ & $\%$ & $\mathbf{N}$ & $\%$ & $\mathbf{N}$ & $\%$ & $\mathbf{N}$ & $\%$ \\
\hline \multicolumn{11}{|l|}{ Religión } \\
\hline $\begin{array}{l}\text { Intento hall ar consuelo en } \\
\text { mi religión o creencias } \\
\text { espirituales }\end{array}$ & 4 & 13,3 & 7 & 23,3 & 7 & 23,3 & 12 & 40,0 & 30 & 100 \\
\hline Rezo o medito & 2 & 6,6 & 7 & 23,3 & 10 & 33,3 & 11 & 36,6 & 30 & 100 \\
\hline Reinterpretación positiva & & & & & & & & & & \\
\hline $\begin{array}{l}\text { Intento ver con otros ojos } \\
\text { mi enfermedad, para hacer } \\
\text { que parezca más positiva }\end{array}$ & 2 & 6,6 & 4 & 13,3 & 3 & 10,0 & 21 & 70,0 & 30 & 100 \\
\hline $\begin{array}{l}\text { Busco algo bueno en mi } \\
\text { enfermedad }\end{array}$ & 5 & 16,6 & 4 & 13,3 & 6 & 20 & 15 & 50,0 & 30 & 100 \\
\hline
\end{tabular}


Tabla 2.2. Estrategias de afrontamiento centrado en la emoción utilizadas por los pacientes con tuberculosis de un centro de salud

\begin{tabular}{|c|c|c|c|c|c|c|c|c|c|c|}
\hline \multirow{2}{*}{$\begin{array}{l}\text { Afrontamiento } \\
\text { centrado en la emoción }\end{array}$} & \multicolumn{2}{|c|}{ Nunca } & \multicolumn{2}{|c|}{ Casi nunca } & \multicolumn{2}{|c|}{ Casi siemp re } & \multicolumn{2}{|c|}{ Siempre } & \multicolumn{2}{|c|}{ Total } \\
\hline & $\mathbf{N}$ & $\%$ & $\mathbf{N}$ & $\%$ & $\mathbf{N}$ & $\%$ & $\mathbf{N}$ & $\%$ & $\mathbf{N}$ & $\%$ \\
\hline \multicolumn{11}{|l|}{ Aceptación } \\
\hline Acepto mi enfermedad & 3 & 10 & 1 & 3,3 & 4 & 13,3 & 22 & 73,3 & 30 & 100 \\
\hline $\begin{array}{l}\text { Aprendo a vivir con } \\
\text { mi enfermedad }\end{array}$ & 2 & 6,6 & 1 & 3,3 & 3 & 10 & 24 & 80 & 30 & 100 \\
\hline
\end{tabular}

Tabla 3. Estrategias de Afrontamiento centrado en la emoción menos funcional utilizadas por los pacientes con tuberculosis de un centro de salud

\begin{tabular}{|c|c|c|c|c|c|c|c|c|c|c|}
\hline \multirow{2}{*}{$\begin{array}{l}\text { Afrontamiento centrado } \\
\text { en la emoción menos } \\
\text { funcional }\end{array}$} & \multicolumn{2}{|c|}{ Nunca } & \multicolumn{2}{|c|}{ Casi nunca } & \multicolumn{2}{|c|}{ Casi siempre } & \multicolumn{2}{|c|}{ Siempre } & \multicolumn{2}{|c|}{ Total } \\
\hline & $\mathbf{N}$ & $\%$ & $\mathbf{N}$ & $\%$ & $\mathbf{N}$ & $\%$ & $\mathbf{N}$ & $\%$ & $\mathbf{N}$ & $\%$ \\
\hline \multicolumn{11}{|l|}{ Negación } \\
\hline $\begin{array}{l}\text { Me digo a mi mismo "no } \\
\text { tengo esta enfermedad" }\end{array}$ & 13 & 43,3 & 2 & 6,6 & 6 & 20,0 & 9 & 30,0 & 30 & 100 \\
\hline $\begin{array}{l}\text { Me ni ego a creer que } \\
\text { estoy enfermo }\end{array}$ & 15 & 50,0 & 3 & 10,0 & 4 & 13,3 & 8 & 26,6 & 30 & 100 \\
\hline \multicolumn{11}{|l|}{ Autodistracción } \\
\hline $\begin{array}{l}\text { Recurro al trabajo o } \\
\text { realizo otras actividades } \\
\text { para apartar la enfermedad } \\
\text { de mi mente } \\
\text { Hago algo para pensar }\end{array}$ & 6 & 20,0 & 1 & 3,3 & 7 & 23,3 & 16 & 53,3 & 30 & 100 \\
\hline $\begin{array}{l}\text { menos en mi enfermedad, } \\
\text { tal como ir al cine o ver la } \\
\text { televisión }\end{array}$ & 4 & 13,3 & 2 & 3,3 & 5 & 16,6 & 19 & 63,3 & 30 & 100 \\
\hline
\end{tabular}

Tabla 3.1. Estrategias de afrontamiento centrado en la emoción menos funcional utilizadas por los pacientes con tuberculosis de un centro de salud

\begin{tabular}{|c|c|c|c|c|c|c|c|c|c|c|}
\hline \multirow{2}{*}{$\begin{array}{l}\text { Afrontamiento } \\
\text { centrado en la emoción } \\
\text { menos funcional }\end{array}$} & \multicolumn{2}{|c|}{ Nunca } & \multicolumn{2}{|c|}{$\begin{array}{c}\text { Casi } \\
\text { nunca }\end{array}$} & \multicolumn{2}{|c|}{$\begin{array}{l}\text { Casi } \\
\text { sempre }\end{array}$} & \multicolumn{2}{|c|}{ Siempre } & \multicolumn{2}{|c|}{ Total } \\
\hline & $\mathbf{N}$ & $\%$ & $\mathbf{N}$ & $\%$ & $\mathbf{N}$ & $\%$ & $\mathbf{N}$ & $\%$ & $\mathbf{N}$ & $\%$ \\
\hline \multicolumn{11}{|l|}{ Autoinculpación } \\
\hline $\begin{array}{l}\text { Me critico a mi } \\
\text { mismo }\end{array}$ & 8 & 26,6 & 7 & 23,3 & 6 & 20 & 9 & 30 & 30 & 100 \\
\hline $\begin{array}{l}\text { Me echo la culpa por } \\
\text { tener esta enfermedad }\end{array}$ & 7 & 23,3 & 5 & 16,6 & 8 & 26,6 & 10 & 33,3 & 30 & 100 \\
\hline \multicolumn{11}{|l|}{ Desconexión conductual } \\
\hline $\begin{array}{l}\text { Renuncio a intentar } \\
\text { ocuparme de mi } \\
\text { enfermedad }\end{array}$ & 12 & 40 & 7 & 23,3 & 4 & 13,3 & 7 & 23,3 & 30 & 100 \\
\hline $\begin{array}{l}\text { Renuncio al intento de } \\
\text { hacer frente a mi } \\
\text { enfermedad }\end{array}$ & 14 & 46,6 & 3 & 10 & 2 & 6,6 & 11 & 36,6 & 30 & 100 \\
\hline
\end{tabular}


Tabla 3.2. Estrategias de Afrontamiento centrado en la emoción menos funcional utilizadas por los pacientes con tuberculosis de un centro de salud

\begin{tabular}{|c|c|c|c|c|c|c|c|c|c|c|}
\hline \multirow{2}{*}{$\begin{array}{l}\text { Afrontamiento centrado } \\
\text { en la emoción menos } \\
\text { funcional }\end{array}$} & \multicolumn{2}{|c|}{ Nunca } & \multicolumn{2}{|c|}{ Casi nunca } & \multicolumn{2}{|c|}{ Casi siempre } & \multicolumn{2}{|c|}{ Siempre } & \multicolumn{2}{|c|}{ Total } \\
\hline & $\mathbf{N}$ & $\%$ & $\mathbf{N}$ & $\%$ & $\mathbf{N}$ & $\%$ & $\mathbf{N}$ & $\%$ & $\mathbf{N}$ & $\%$ \\
\hline \multicolumn{11}{|l|}{ Desahogo } \\
\hline $\begin{array}{l}\text { Digo cosas para liberar } \\
\text { sentimientos } \\
\text { desagradables acerca de } \\
\text { mi enfermedad }\end{array}$ & 12 & 40 & 8 & 26,6 & 7 & 23,3 & 3 & 10 & 30 & 100 \\
\hline $\begin{array}{l}\text { Expreso mis sentimientos } \\
\text { negativos acerca de mi } \\
\text { enfermedad }\end{array}$ & 12 & 40 & 5 & 16,6 & 7 & 23,3 & 6 & 20 & 30 & 100 \\
\hline \multicolumn{11}{|l|}{ Uso de sustancias } \\
\hline $\begin{array}{l}\text { Utilizo alcohol u otras } \\
\text { drogas para hacerme } \\
\text { sentir mejor }\end{array}$ & 23 & 76,6 & 4 & 13,3 & 2 & 6,6 & 1 & 3,3 & 30 & 100 \\
\hline $\begin{array}{l}\text { Utilizo alcohol u otras } \\
\text { drogas para ayudarme a } \\
\text { superar mi enfermedad }\end{array}$ & 25 & 83,3 & 2 & 6,6 & 2 & 6,6 & 1 & 3,3 & 30 & 100 \\
\hline
\end{tabular}

Tabla 3.3. Estrategias de afrontamiento centrado en la emoción menos funcional utilizadas por los pacientes con tuberculosis de un centro de salud

\begin{tabular}{|c|c|c|c|c|c|c|c|c|c|c|}
\hline \multirow{2}{*}{$\begin{array}{l}\text { Afrontamiento centrado en } \\
\text { la emoción menos funcional }\end{array}$} & \multicolumn{2}{|c|}{ Nunca } & \multicolumn{2}{|c|}{ Casi nunca } & \multicolumn{2}{|c|}{ Casi siempre } & \multicolumn{2}{|c|}{ Siempre } & \multicolumn{2}{|c|}{ Total } \\
\hline & $\mathbf{N}$ & $\%$ & $\mathbf{N}$ & $\%$ & $\mathbf{N}$ & $\%$ & $\mathbf{N}$ & $\%$ & $\mathbf{N}$ & $\%$ \\
\hline \multicolumn{11}{|l|}{ Humor } \\
\hline $\begin{array}{l}\text { Hago bromas sobre mi } \\
\text { enfermedad }\end{array}$ & 19 & 63,3 & 5 & 16,6 & 2 & 6,6 & 4 & 13,3 & 30 & 100 \\
\hline Merio de mi enfermedad & 20 & 66,6 & 4 & 13,3 & 1 & 3,3 & 5 & 16,6 & 30 & 100 \\
\hline
\end{tabular}

\section{DISCUSIÓN}

En el 2011; 8,7 millones de personas enfermaron de tuberculosis y 1,4 millones murieron por esta causa, la tuberculosis es considerada uno de los principales problemas de salud pública más prevalente y de gran impacto a nivel físico, psicológico, social y económico (1). El impacto de recibir un diagnóstico positivo de tuberculosis se manifiesta como una amenaza y constituye un evento estresante que no solo depende del diagnóstico, sino que existen variables individuales que influyen en la duración de dicho impacto tales como: la edad, el sexo, las creen- cias previas sobre la enfermedad y los tratamientos que se tengan (8). Además, altera significativamente el funcionamiento individual y se relaciona a un conjunto de atributos o juicios sociales mayormente desacreditantes, 1lamados estigmas (9), que fomentan actitudes discriminatorias y repercuten ostensiblemente las interacciones y roles de la persona que la padece.

La tuberculosis (TB) está asociada frecuentemente a la existencia de trastornos emocionales y de conducta; asimismo, la falta de conciencia de enfermedad, soporte familiar y/o social, como el consumo de sustancias 
psicoactivas, son factores de riesgo no farmacológicos que pueden dificultar la culminación del tratamiento. El presente estudio de investigación se realizó en el Centro de Salud «Santa Rosa», en una población de 30 pacientes teniendo como objetivo identificar las estrategias de afrontamiento frente al diagnóstico de tuberculosis utilizadas por ellos.

En cuanto a los datos sociodemográficos el $63,3 \%$ de los pacientes fueron de sexo masculino y $36,6 \%$ de sexo femenino. Se observa así que los hombres tienden a contraer la tuberculosis más que las mujeres. De la misma manera, diversos estudios desarrollados a escala mundial expresan que la tuberculosis afecta más a los hombres que a las mujeres (10). El hecho de que los índices de notificación de tuberculosis sean más elevados en los hombres puede obedecer, en parte, a diferencias epidemiológicas (en cuanto a exposición, riesgo de infección y progresión desde el estadio de infección al de enfermedad) (8) ya que el hombre, al ser proveedor económico del hogar, es más vulnerable a exponerse a situaciones que implica el riesgo de infección de TB.

También se encontró que el $60 \%$ tiene entre 18 - 30 años y $40 \%$ tiene entre 31 - 60 años, dato que muestra como la tuberculosis tiene una distribución mayor en la población adulto joven. El MINSA se manifiesta ante este fenómeno afirmando que la tuberculosis es una enfermedad social totalmente curable que afecta a la población económicamente activa, siendo los grupos de edad más afectados los comprendidos entre los 15 y 54 años, resalta que los adolescentes y adultos mayores constituyen alrededor de la tercera parte del total de casos (1).

Otro aspecto interesante es el referente al nivel de instrucción de la población estudiada. Los resultados muestran que el $70 \%$ cuenta con educación secundaria, dato importante que considerar ya que el nivel escolar es una característica importante en los grupos humanos puesto que condiciona la ocupación laboral, los ingresos económicos de las personas y, por lo tanto, los recursos de esta para satisfacer sus necesidades. Esta información la podemos corroborar con el informe del INEI, donde se muestra que a nivel nacional, prevalece la enseñanza secundaria como el mayor nivel alcanzado por los jefes/jefas de hogares. Así mismo, expresa que el nivel educacional establece diferencias entre personas en términos de acceso a la información, perspectivas y posibilidades de beneficiarse de nuevos conocimientos; constituye una premisa que condiciona de modo regular la ocupación laboral y un componente determinante de la cultura y la educación, además que permite comprender y enfrentar mejor los fenómenos sociales o enfermedades que se puedan suscitar (9).

La carencia de una entrada financiera conduce a la pobreza y problemas de salud como consecuencia de un progreso desigual. Cabe mencionar que en la presente investigación no se obtuvo información de los ingresos económicos de los pacientes; sin embargo, es importante considerar que la población pertenece a San Juan de Lurigancho, distrito que según el INEI se encuentra en situación pobre, categorizado como zona marginal. Así mismo, es el distrito con mayor número de habitantes, que resulta un incremento de población con problemas de pobreza y hacinamiento (9).

Con respecto a las estrategias utilizadas por los pacientes, el presente estudio encontró que el afrontamiento activo fue utilizado por un $86,6 \%$ de los pacientes. Esta estrategia, definida por Carver en 1989, requiere que el sujeto redoble sus esfuerzos y que de a poco trate de resolver el problema, iniciando acciones directas e incrementando sus propios esfuerzos para eliminar o reducir el estresor, en este caso la presencia de la enfermedad (11). Es por ello, que el solo hecho de afrontar es indicativo de una conducta activa, que se asocia al afrontamiento centrado en la solución del problema ya que favorece a la adaptación positiva del paciente, a su condición médica y optimiza la adhesión al tratamiento, dando a entender que los pacientes ejecutan lo recomendado por los profesionales de la salud que los atienden.

Por lo que se puede concluir que la mayoría de pacientes, a pesar del importante desajuste que sufren, estos dejan de hacer otras actividades para concentrarse en su enfermedad, lo cual puede repercutir en la adopción de conductas saludables contribuyendo al proceso de adaptación y recuperación del paciente, así como en su calidad de vida. Así mismo, el afrontamiento centrado en el problema parece estar asociados a resultados psicológicos más positivos, al favorecer la adaptación a la nueva condición de enfermo (12). Así mismo, la «planificación» fue utilizada por un $60 \%$ de los pacientes. Esto también se puede evidenciar en los resultados de Delgado Mollo, quien dirigió un estudio que tuvo como objetivo identificar la relación que existe entre las estrategias de afrontamiento más utilizadas de las mujeres con cáncer de mama y algunas características sociodemográficas, donde obtuvo que la «planificación» fue utilizada en un 78,57\% (13). El hecho de que se encuentren resultados similares en poblaciones diferentes se puede sustentar de que en ambos estudios los pacientes tienen más de 1 mes de diagnosticada la enfermedad, estas son crónicas e implican un tratamiento 
prolongado, reflejando la necesidad del paciente de organizar estrategias de acción, analizando y planificando que pasos tomar y establecer cuál es la mejor forma de manejar el problema.

Es importante mencionar que en el estudio realizado por Vargas J (13), observó que en los primeros años de recibir el diagnóstico los pacientes muestran más capacidad para hacer planes sobre su persona, lo cual coincide con los resultados encontrados en este estudio ya que el 56,6\% de los pacientes diagnosticados de tuberculosis tienen más de 1 mes de haber sido diagnosticados. En las estrategias de afrontamiento centradas en la emoción, las acciones de los pacientes están dirigidas a disminuir o eliminar las reacciones emocionales desencadenadas por la fuente estresante (tuberculosis) (7); por lo tanto, el paciente, al evaluar la situación como inflexible e imposible de cambiar, va a esforzare por sentirse bien emocionalmente tratando de encontrar un equilibrio psicológico que lo ayude a enfrentar la situación.

En esta categoría la estrategia de afrontamiento «aceptación» fue usada por un $80 \%$ de la población, mientras que Pillaca encontró que esta misma estrategia se encuentra ausente en el $57 \%$ de los pacientes, y que la religión se encuentra presente en un $57 \%$ de la población (12). La aceptación implica aceptar la realidad como un hecho con el que se tendrá que convivir, ya que no se podrá cambiar (14). Esto quiere decir que los pacientes que usan esta estrategia son conscientes de lo que implica tener una enfermedad crónica; por lo tanto, van a tener los recursos necesarios para lidiar con la sintomatología de la enfermedad y el tratamiento. De la misma manera, van a ser adherentes al tratamiento, ya que a pesar de los síntomas adversos que experimentan, reconocen las ventajas a futuro que pueden tener.

La diferencia de estos resultados posiblemente se deba a que Pillaca (12) en su estudio incluye a pacientes con TBC MDR, mientras que en esta investigación solo se incluye pacientes con TBC sensible. Este aspecto es importante, ya que cierta cantidad de la población presenta un tipo de TBC con una evolución y tratamiento distinto, donde las probabilidades de curarse son menores y, en muchos casos, llegan a ser XDR aumentando las posibilidades de morir; en estos casos, las creencias religiosas le permiten al paciente darle un sentido a la vida, a la enfermedad y a su posible desenlace.

Por otro lado, se encontró en este estudio que el 23,3\% no hace uso del apoyo instrumental ni emocional. Es probable que esto se deba a que el perfil sociodemográfico responde a un mayor porcentaje de pacientes adultos jóvenes y solteros ya que esta etapa del desarrollo humano se caracteriza por cambios drásticos en las relaciones personales así como un tiempo más limitado para estar con los amigos o la familia. El tener una enfermedad y no contar con el apoyo de los pares, compañeros románticos o la misma familia puede aumentar el desajuste emocional.

Para Enfermería el apoyo instrumental y uso de apoyo emocional son dos estrategias que implican un sistema con un patrón de vínculos sociales continuos e intermitentes que van a desempeñar una parte significativa en el mantenimiento de la integridad física y psicológica de los pacientes con tuberculosis a fin de que continúen con el tratamiento y recuperen su estado de salud (15). Es importante destacar el papel que cumple la red de apoyo social que poseen las personas (pareja, familiares, amigos, profesionales de la salud) ya que es fundamental para desarrollar un mejor nivel de afrontamiento dado que esto hace referencia a sentirse querido, protegido y valorado por personas cercanas en quien poder confiar (7).

Referente a las otras estrategias de afrontamiento, también llamadas centradas en la emoción menos funcional, están definidas como el intento que realiza la persona para encontrar significado al suceso y valorarlo de manera que resulte menos desagradable para esta (16); sin embargo, la persona realiza diversas acciones a fin de no pensar en el problema, y lo aleja de la realidad que en muchas veces puede llevarlo a tener conductas que no favorecerán a afrontar el estresor. La estrategia de afrontamiento «autodistracción» fue utilizada por un $63,3 \%$ de la población, resultados similares se obtuvieron en el estudio realizado por Pillaca el cual obtuvo que en el $60 \%$ de su población está presente esta misma estrategia (12).

La «autodistracción» implica concentrarse en otras acciones o proyectos, intentando distraerse con otras actividades, para tratar de no concentrarse en el estresor (8). Es una de las maneras de afrontar el estrés durante la enfermedad cuando se observa que quejarse inquieta a familiares y amigos, o bien cuando la realidad es dura y difícil de soportar. El utilizar esta estrategia es un resultado esperable, puede ser positiva por un lado, si es que se busca por ciertos momentos alejar el estresor; sin embargo, puede llegar a ser negativa cuando se necesite encarar directamente la situación, perjudicando así su estado de salud y su recuperación (16). Por otro lado, la estrategia «uso de sustancias» fue utilizada por el 3,3\% de los pacientes. Se considera que este resultado es un buen indicador ya que el uso de sustancias puede dar temporal- 
mente cierta tranquilidad, pero posteriormente, puede interferir en la adaptación a la enfermedad. Es una estrategia de afrontamiento que puede contribuir al abandono del tratamiento o empeoramiento del estado de salud, cuando el paciente no asume conductas de autocuidado de la salud, impidiendo así que se logre el propósito de garantizar la adherencia al tratamiento. Es por ello que Viviano, la agrupa dentro de un grupo llamado estrategias centradas en la emoción menos funcional; que son asociadas con bajos niveles de bienestar y que en este estudio no proporcionan una adecuada adherencia y recuperación (16).

Para terminar, los profesionales de enfermería como personal de salud que trabajan directamente y exclusivamente en el PCT, tienen el compromiso de ayudar a cada paciente de manera personalizada, respetando y aplicando los principios y valores humanos (17), no solo a nivel físico sino y sobre todo a nivel psicológico, que al fin y al cabo esto es lo que a muchos de los pacientes los sostiene $\mathrm{y}$ fortalece para seguir adelante con esta enfermedad.

Se sugiere al personal de Enfermería desarrollar técnicas de intervención orientadas a proporcionar recursos y estrategias a los pacientes con TBC, para ayudar a minimizar el impacto de la enfermedad, así como sus secuelas psicológicas, sociales y físicas, logrando así una adecuada adherencia y su pronta recuperación y fomentar el uso de las estrategias centradas en el problema, los pacientes que asisten a la ESN-PCT del Centro de Salud Santa Rosa utilizan en mayor porcentaje el afrontamiento activo.

\section{REFERENCIAS BIBLIOGRÁFICAS}

1. Ministerio de Salud del Perú. [Internet] 2010 (citado 19 Jul 2012). Disponible en: http://www.minsa.gob.pe/

2. Dirección de Salud (DISA) IV Lima Este del Ministerio de Salud. [internet] 2009 (citado 15 Jul del 2012). Disponible en http://www.disalimasur.gob.pe/ DISA_HomeM1.aspx

3. Arrossi S, Herrero M, Greco A, Ramos S. Factores predictivos de la no adherencia al tratamiento de la tuberculosis en municipios del Área Metropolitana de Buenos Aires, Argentina. Salud colectiva [Internet] 2012 (citado 18 Dic 2014); 8(Supl 1): S65-S76. Disponible en: http://www.scielo.org.ar/pdf/sc/v8s1/ v8s1a12.pdf

4. Oldham J, Skosol A, Bender D. Tratado de los trastornos de la personalidad. 1era Ed. España: Elsevier Doyma; 2007.

5. Felipe E, et al.. Estrategias de afrontamiento del estrés y conducta interpersonal. International Journal of Psychology Therapy. España. [Internet] 2010 (citado 18 Dic 2014); 10 (2) 245-257. Disponible en: http://www.ijpsy.com/volumen $10 /$ num $2 / 260 /$ estrategias-de-afrontamiento-del-estrs-ES.pdf

6. Musayón Oblitas F, et al. El rol de la enfermería en el control de la Tuberculosis: Una discusión desde la perspectiva de la equidad. Perú. [Internet] 2010 (citado 22 Jul 2012); 18 (1). Disponible en: http:// www.scielo.br/pdf/rlae/v18n1/es_20.pdf

7. Norma técnica de salud para el control de la tuberculosis: BSCDE. [Internet] 2010 (citado $18 \mathrm{Jul}$ 2012). Disponible en: http://www.bvsde.paho.org/ texcom/sct/048026.pdf

8. Aparicio A, et al. Estrategias de afrontamiento del cuidador primario del paciente con trastorno esquizofrénico. Perú. Universidad Peruana Cayetano Heredia, 2010.

9. Bermejo Nava C. Neumología: Epidemiología de la tuberculosis. [Internet] 2008 (citado $10 \mathrm{Jul} 2012$ ); vol. 30(1). Disponible en: http://scielo.isciii.es/pdf/asisna/ v30s2/original1.pdf

10. Morán $\mathrm{C}$, et al. Cope-28 Un análisis psicométrico de la versión en español del Brief COPE: Universitas Psychologica. [Internet] Ene 2010 (citado 5 Jul 2012); 9 (2), 543-552. Disponible en: http:// redalyc.uaemex.mx/src/inicio/ArtPdfRed.jsp? $\mathrm{iCve}=64716832020$

11. Instituto Nacional de Estadística e Informática. [Internet] 2013 (citado 15 Mar 2014). Disponible en: http://www.inei.gob.pe/

12. Pillaca Gonzales H. Estrategias de afrontamiento del paciente que asiste a la ESN-PCT en el Centro de Salud Max Arias Schreiber. Perú. Universidad Nacional Mayor de San Marcos; 2013.

13. Vargas Mendoza J, et al. Estrategias de afrontamiento del SIDA en pacientes diagnosticados como seropositivos. México. [Internet] 2009 (citado $20 \mathrm{Jul}$ 2012). Disponible en: http://www.uv.mx/psicysalud/ psicysalud-19-2/19-2/Jaime-Ernesto-VargasMendoza.pdf

14. Control mundial de la tuberculosis - Informe. [Internet] 2013 (citado 15 Mar 2014). Disponible en: http://www.who.int/tb/publications/global_report/es/

15. Delgado Mollo A, et al. Relación entre la estrategia de afrontamiento más utilizadas por las mujeres con cáncer de mama y algunas características sociodemográficas. Lima - Perú. Universidad Peruana Cayetano Heredia; 2005.

16. Díaz Viviano L, et al. Afrontamiento del cuidador primario de un adulto mayor con depresión del programa de prestación de atención domiciliaria en el 
distrito de Comas. Perú. Universidad Peruana Cayetano Heredia; 2011.

17. Felipe Castaño E, et al. Estrategias de afrontamiento del estrés y estilos de conducta interpersonal. España; 2011

\section{Correspondencia}

Valeria Fernández Cruzado

Universidad Peruana Cayetano Heredia

Dirección: Av. Honorio Delgado 430, Urb. Ingeniería, Lima - 31

Correo electrónico: valeria.fernandez.c@upch.pe

Forma de citar este artículo: Belito-de la Cruz H; FernándezCruzado V; Sánchez-Gutiérrez S; Farro-Peña G. Estrategias de afrontamiento utilizadas por los pacientes con tuberculosis de un centro de salud. Rev enferm Herediana. 2014;7(2):69-78. 